\title{
Optimizing Performance of a Fiber Optic Sensor Interrogator by Time of Flight Measurement
}

\author{
Plattner M. P., Hirth F., Müller M. S., Hoffmann L., Buck T. C., Koch A. W. \\ Technische Universität München \\ Lehrstuhl für Messsystem- und Sensortechnik \\ Theresienstr. 90 / N5 \\ 80333 München
}

\begin{abstract}
For the purpose of structure and temperature monitoring of Ariane launchers, a new fiber optic sensor interrogation system is developed by our institute. It is based on a monolithic, modulated-grating laser diode that is capable of switching between any two output wavelengths within less than ten nanoseconds. This makes the system very versatile since different sensors within the connected measurement channels can be read out in arbitrary sequence. Therefore sampling rates of single sensors can be adapted according to their application independently.

The connection between spectral and time domain during read out of sensors is clarified. Performance optimization due to "Time of Flight" measurement and its consequences for different sample sequences are described. Issues that occur because of switching between different output wavelenghts during operation of the system are described in detail and solutions based on software algorithms are presented.
\end{abstract}

\section{INTRODUCTION}

Fiber optic interrogation systems are usually implemented as one of two well known configurations: Spectrometer based systems utilize a broadband illumination and detect the actual wavelength of the sensor by spatial division multiplexing. On the other hand, systems based on tuneable lasers sweep through the wavelength spectrum and evaluate the transmitted (or reflected) intensities, depending on the laser's output wavelength. Our system is part of the second group and is developed within the project "Structural Monitoring of Ariane Launchers using Fiber Optic Sensing" funded by "European Space Agency" [1]. The Ariane launcher is currently monitored by hundreds of electric sensors during test and qualification. Fiber optic sensing is regarded as a potential technique to overcome limitations of recent Ariane monitoring systems. These limitations include cumbersome application of sensors and harness as well as a very limited degree of distributed sensing capability. Various advantages including high degree of multiplexing, distributed sensing capability, immunity to electromagnetic interference, etc. can be exploited by the use of fiber optic sensing [3]. However, when it comes to the number of sensors, versatility in application, cost, vibration hardness or installation space, shortcomings in the current generation of these measurement systems are obvious. A dedicated measurement system has to be developed and investigated during the project. Instruments based on tuneable lasers are established devices for demodulation of fiber optic sensors [2]. These state-of-the-art systems built on tuneable laser sources often use free beam setups for wavelength tuning. Thus they are sensitive to environmental influences like vibration or temperature impacts. Our new sensor interrogation system is based on an electro-optically tuneable laser diode. It is a monolithic device and therefore insensitive to vibration loads. Compared to already existing interrogation systems for fiber optic sensors, our interrogator allows us to enhance measurement capabilities. It is capable of measuring various kinds of optical fiber sensors including sensors based on Bragg gratings, Brillouin scattering and Fabry-Perot interferometers. By also using fiber coupled photo detectors for input intensity measurement, none of the subcomponents is sensitive to environment, provided that the laser diode is adequately temperature controlled.

\section{MEASUREMENT SETUP}

Our measurement setup is shown in figure 1. Three optical fibers can be connected to the interrogator. Up to ten sensors inside each fiber can be mounted. Within this article, only sensors based on fiber Bragg gratings (FBG) are considered. The spectral answer of a FBG sensor is calculated by a centroid algorithm. Thereto ten spectral points are sampled for each sensor [4].

Core of the system is a modulated-grating tuneable laser diode. The non-transmissive mirror of the optical resonator is split into two end reflectors realized as grating structures. The wavelength is tuned by three input control currents fed into the gratings and into a common phase region of the resonators. The output spectrum of the laser diode is not continuous but discrete wherein consecutive wavelengths have a mean distance of about $4 \mathrm{pm}$. The output wavelength is tuneable in the wavelength range from $1527 \mathrm{~nm}$ to $1568 \mathrm{~nm}$ by adjusting the input current triplet appropriately. This yields more than $10^{4}$ 
different wavelengths. The maximum switching frequency of the laser is in the $\mathrm{GHz}$ region and within our setup limited by the performance of the electronics.

The control unit accommodates DAC modules for adjusting the output wavelength of the laser diode and $A D C$ modules for digitizing the measured analog intensity values. Being able to adjust three output currents and to digitize three input signals in parallel, a FPGA controls ADC and DAC modules. Additionally a DSP is implemented inside the control unit that executes measurement algorithms.

Fiber couplers distribute the laser light to all sensor fibers and guide the reflected sensing signals to the detection unit. Intensity values are detected by photo detectors, amplified and transferred to the controller unit.

The number of measurement channels is limited to three within our setup. This is due to the purchased hardware configuration, which does not support a bigger number of ADC modules. In principle the system is scalable to a higher number of channels by using a different read-out hardware. Therefore all considerations within this article are kept in general and are therefore not limited to our current hardware configuration. This permits adaptablility to further developement without the need for recalculation.

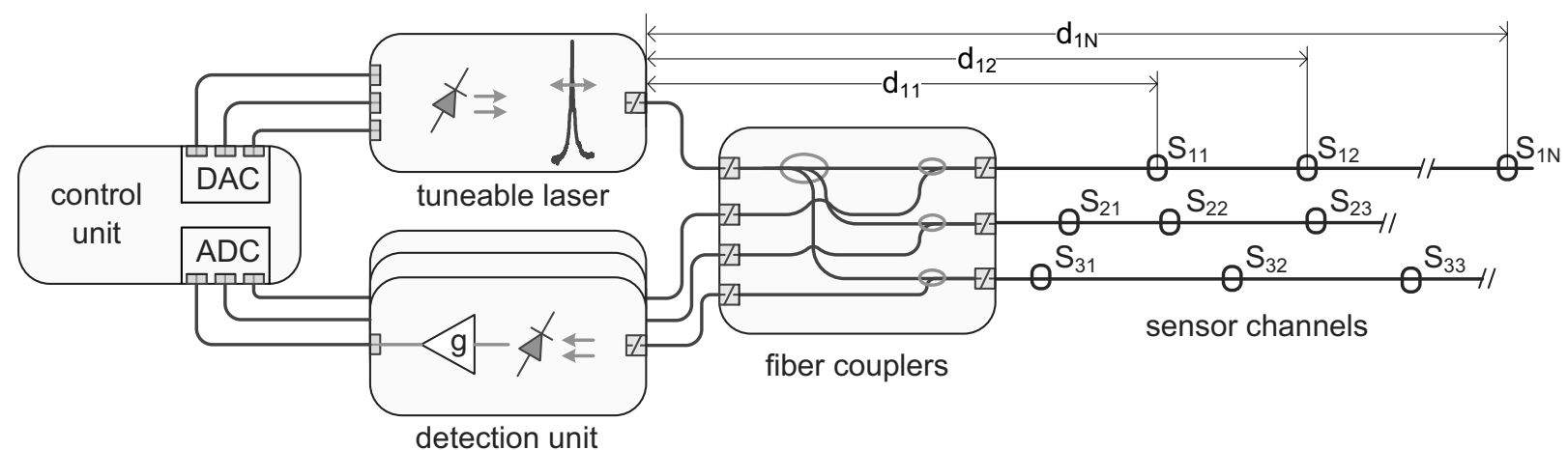

Figure 1: The fiber optic interrogation unit. The control unit adjusts the output wavelength of the tuneable laser diode. The sensor signal intensities are measured by a detection unit. Evaluation of the measurement values is carried out by the control unit. Different sensor distances $d_{i}$ are illustrated.

\section{TIME OF FLIGHT MEASUREMENT CONCEPT}

Since this interrogation system is built on the tuneable laser diode, changing between any two output wavelengths within the entire spectrum of the laser diode takes the same amount of time (less than $10 \mathrm{~ns})$. This enables a high versatility, i.e. it is possible to jump to different parts of the spectrum for reading out only single sensors, not the entire group of sensors inside the channel.

Consequently the sampling rate of sensors can be adapted to their application independently. If for instance a temperature sensor monitors a slowly varying part of the Ariane launcher, it can be read out using a slow measurement rate. High sampling rates can be chosen for important sensors that are needed to prevent the launcher from structural damages.

"Time of Flight" (ToF) is defined as the travel time of a sensing light pulse. Since quantities and positions of the sensors inside one channel vary for each measurement application and for each sensor channel, the ToF for each sensor has to be evaluated prior to the operation of the system.

According to figure $1, T o F_{i}$ of sensor $i$ is dependent on the distance $d_{i}$ between interrogator and sensor. Assuming a refractive index $n$ of the fiber material, the ToF results in:

$$
T o F_{i}=\frac{2 d_{i} \cdot n}{c}
$$

wherein $c$ stands for the speed of light in vacuum. Additionally a constant ToF value ( $\Delta$ ToF) has to be added to all calculated ToF values. This offset is due to extra fiber length between laser diode (LD), photo detectors (PD) and the measurement channel connectors. Assuming an extra length of $1 \mathrm{~m}$ results in a constant ToF offset of $\Delta T o F \approx 10 n s$.

A maximum number of $M$ sensor fibers (measurement channels) can be connected to the interrogator. Within each channel a number of $N_{m}$ sensors are implemented. Therefore the ToF values are indicated by two digits. The first one stands for the channel, the second one indicates the position of the sensor within the sensor fiber. See Figure 2 for illustration. 


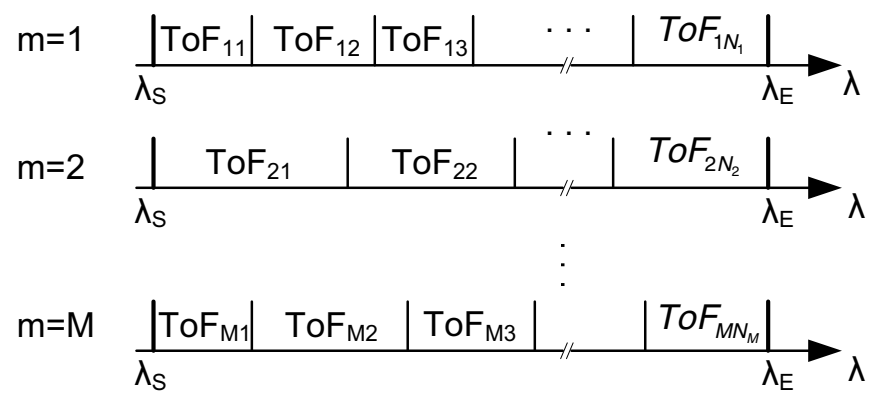

Figure 2: $M$ fibers are connected to the interrogator. The spectrum of the laser is divided into $N_{m}$ regions for each measurement channel. The ascending order of all ToF values in the spectral domain shown here only occurs if the sensors inside the fibers are implemented in ascending wavelength order as the distance to the interrogator increases. This is usually not the case but is chosen here for better visualization.

The number of sensors $\mathrm{N}_{\mathrm{m}}$ implemented in one fiber may vary from channel to channel. Equal distances between interrogator and sensors of different channels result in equal ToF values. This does not effect the measurement concept.

The above described advantage of being able to switch to any wavelength entails the drawback, that the control unit is forced to take care of the timing. If one sampling pulse has been sent to sensor $i$, related intensities can be measured by the detection unit only after a delay time equal to $T o F_{i}$. Therefore all ToF values for all $N_{m}$ sensors inside one sensor fiber need to be calculated by formula (1).

The overall measurement frequency of our interrogation system is limited by the sampling rate $f_{A D C}$ of the ADC module. Due to this quantization, our interrogation system can only handle ToF values as muliples of $T_{A D C}=1 / f_{A D C}$. The interrogator emits a pulse and waits a predetermined number of clock cycles of $f_{A D C}$ before it measures the according photo detector input. Therefore calculated ToF values have to be converted to multiples of $T_{A D C}$ by the algorithm shown on the righthand side of figure 3 .

In order to meet the sampling theorem, the output duty cycle $T_{P}$ of the laser must be longer than $T_{A D C}$ (see figure 2). Since the sampling rate $f_{D A C}$ of our DAC modules is two times $f_{A D C}$ we set the duty cycle to a constant value of $T_{P}=1.5 \cdot T_{A D C}$.

Within our system the maximum ADC sampling rate is $25 \mathrm{MHz}$. Assuming that a single sensor has to be evaluated by a centroid algorithm based on 10 sample points, ten measurements are required. This takes a time of $T_{s}=10 \cdot 2 \cdot T_{A D C}=0.8 \mu \mathrm{s}$ for a single sensor measurement, for $T_{0}=2 \cdot T_{A D C}$. The interrogation system, if operated with one single sensor only, has therefore a maximum measurement frequency of $1.25 \mathrm{MHz}$.

a)

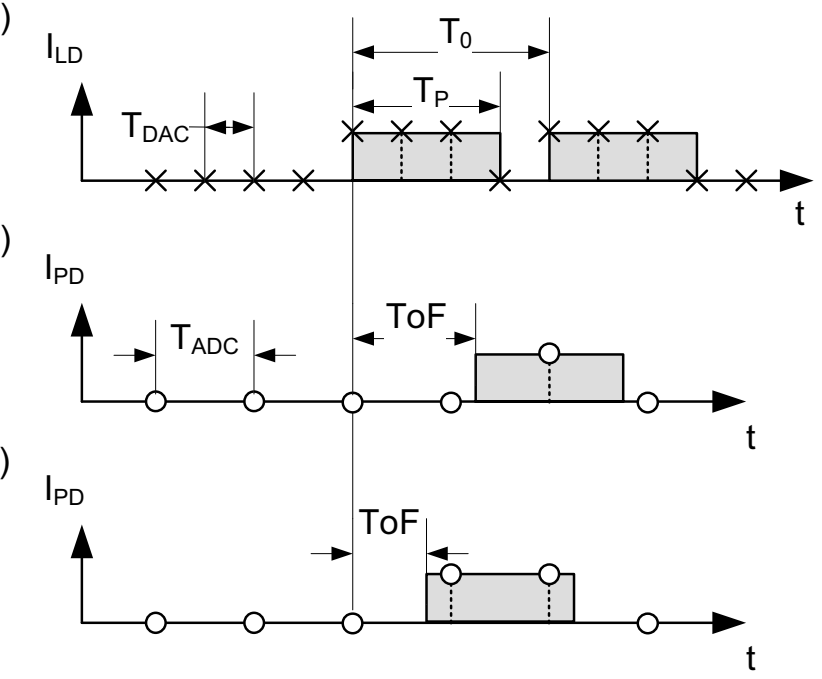

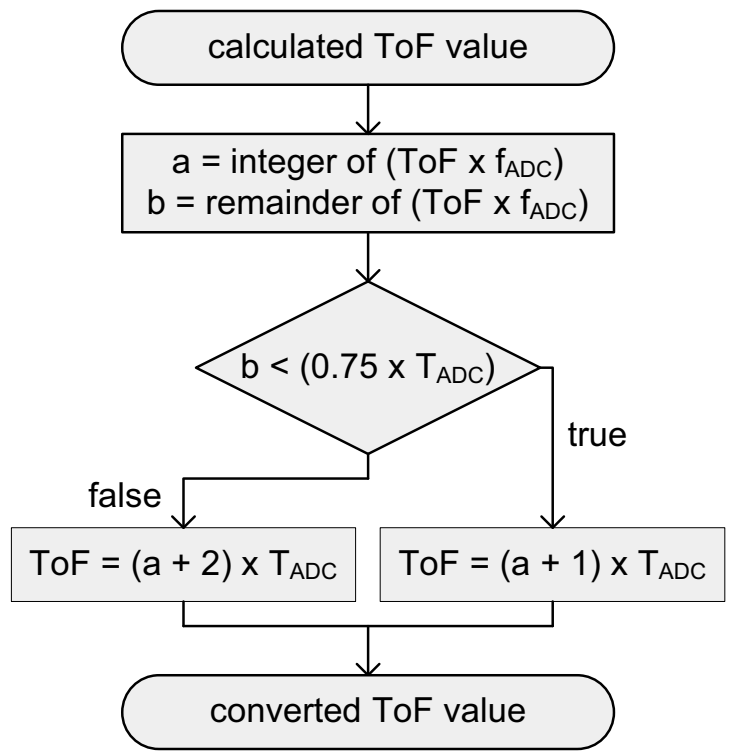

Figure 3: a) The output $I_{L D}$ of the laser diode is controlled by the DAC modules. In order to get at least one sampling point at the ADC input, $T_{P}$ is set to a constant value of $1.5 \cdot T_{A D C}$. Dependent on the ToF of the sensor, one intensity value (b) or two intensity values (c) are sampled. The ToF values are converted to multiples of $T_{A D C}$ by the algorithm shown at the righthand side of the figure. 
It must be ensured by the design of the measurement application, that sensors inside one measurement channel may not be able to overlap spectrally. Each sensor has to be restricted to its spectral range wherein it varies due to changes of the measured quantity. Dependent on the number $N_{m}$ of sensors inside a channel and the associated measurement ranges of each sensor, the entire spectrum of the laser is divided into $N_{m}$ regions of different ranges according to Figure 2

If a sensor channel holds $N_{m}$ sensors, it has to be dealt with $N_{m}$ different ToF values. By wavelength switching within the entire spectrum of the laser, $N$ ! different sequences and $N \times(N-1)$ different transitions from one ToF region to another one are possible.

One major problem of the measurement concept is a possible overlap of incoming pulses at the photodetector (see Figure 4). This happens e.g. if two sensors inside the same channel are read out in an inappropriate order. As long as all sensors are read out in the order of rising ToF values, overlapping of pulses does not occur. If sensors have to be read out in reverse order and overlap, a delay time of $\Delta t$ according to formula (2) needs to be inserted.

$$
\Delta t=\left(T o F_{m n}+T_{P}\right)-\left(T o F_{m(n+1)}+T_{0}\right)
$$

a)

b)

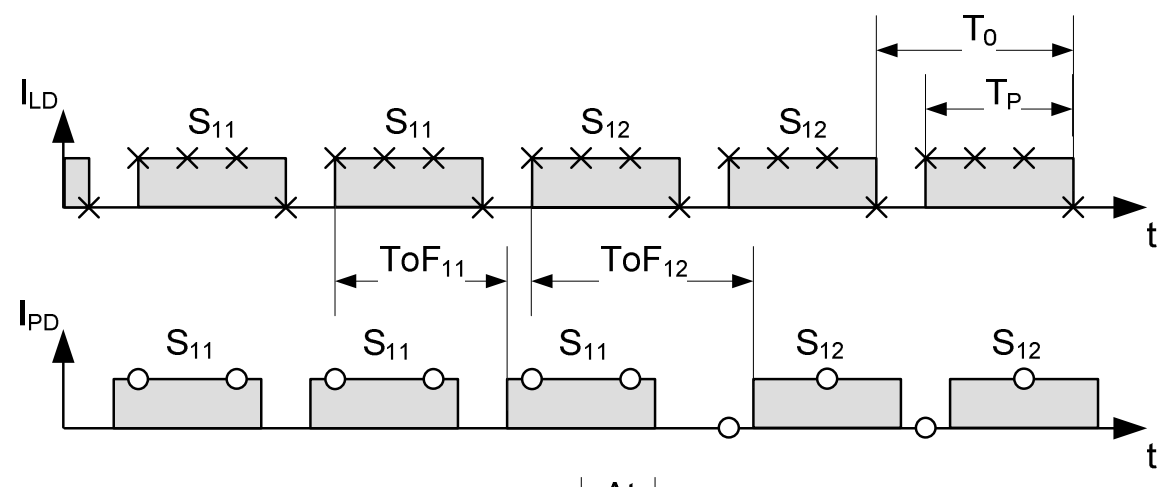

c)

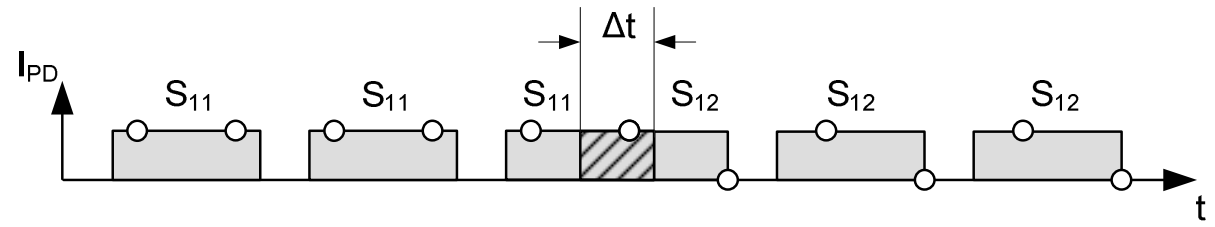

Figure 4: a) Read out of two successive sensors inside one measurement channel. a) The output of the laser diode switches between the wavelengths of sensor $S_{11}$ and $S_{12}$. b) As long as the first sensor has a shorter ToF than the second one, no overlapping can occur at the photo detector. c) If the first sensor has a longer ToF than the second sensor, the gap between successive sensor signals at the photo detector decreases. In order to avoid overlapping, a delay time of $\Delta \mathrm{t}$ has to be inserted.

Considering a number of $M$ sensor channels complicates the overlapping problem. Since every fiber contains $N_{m}$ different sensors, two or more sensors of different channels can overlap spectrally (see Figure 5). In order to avoid any overlap at the detectors, each channel has to be read out in the order of rising ToF values. Parallel read out of the two channels shown in Figure 5 would therefore result in a measurement sequence of $S_{21} \rightarrow S_{11} \rightarrow S_{12} \rightarrow S_{22} \rightarrow S_{23}$. On the righthand side of Figure 5 the spectral positions of the sensors are shown. Sensor $S_{12}$ spectrally overlaps with sensor $S_{23}$. Interrogating all sensors in the above mentioned order could, in this example, result in an overlap of sensor pulses $S_{22}$ and $S_{23}$ at the photo detector of channel two.

One possible solutions to this problem is to design the measurement system in a way that sensors of different channels are not able to overlap. This, however, would restrict the high versatility of the interrogation system. Moreover the adaptabiltiy to existing applications, wherein for example sensor fibers are embedded in composite material, would become lost.

A solution that can be implemented by software engineering is the calculation of a delay time $\Delta t$ according to formula (2) and its insertion between the consecutive pulses that read out $S_{23}$ and $S_{22}$ to avoid overlapping. 
$m=1$

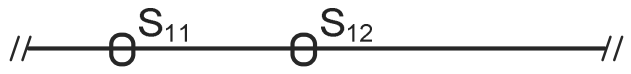

$\mathrm{m}=2$

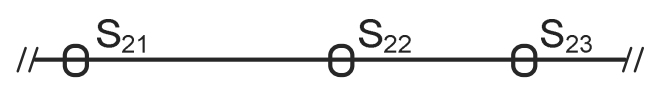

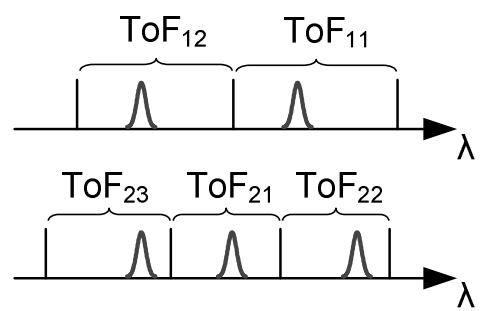

Figure 5: Two measurement channels are considered. Reading out the sensors in the order of increasing ToF values fails, because sensor $S_{12}$ overlaps spectrally with sensor $S_{23}$.

\section{CONCLUSION}

The concepts of "Time of Flight" measurement and the issues concerning different measurement szenarios using our interrogation system have been discussed in detail. The capability of the system has been shown and new ways of measurement have been demonstrated. All considerations have been kept in general in order to enable easy adaptation to similar measurement arrangements.

\section{REFERENCES}

[1] European Space Agency, "Fiber Optic Sensors for Structural Monitoring of the Ariane Launcher", Statement of Work, contract number 1-5353/07/NL/CP, 2007.

[2] Measures R. M., Ohn M. M., Huang S. Y., Bigue J., Fan N. Y., "Tunable laser demodulation of various fiber Bragg grating sensing modalities," Smart Materials and Structures 7 (2), p.237, 1998.

[3] Hoffmann L.; Müller M.S.; Krämer S.; Giebel M.; Schwotzer G.; Wieduwilt T., „Applications of fiber optic temperature measurement”, Proc. Estonian Acad. Sci. Eng., 13(4), pp. 363-378, 2007.

[4] Plattner M. P., Hirth F., Müller M. S., Hoffman L., Buck T. C., Koch A. W. „A versatile fiber optic sensor interrogation system for the ariane launcher based on an electro-optically tuneable laser diode" Proc. Int. Conf. on Space Optics 2008. 